\title{
Coordinated voltage control scheme for distribution grid with on-load tap-changer and distributed energy resources in a market context
}

Han, Xue; Bindner, Henrik W.; Mehmedalic, Jasmin; Tackie, David Victor

Published in:

Proceedings of 2015 41th IEEE IES Annual Conference (IECON'15)

Publication date:

2015

Document Version

Peer reviewed version

Link back to DTU Orbit

Citation (APA):

Han, X., Bindner, H. W., Mehmedalic, J., \& Tackie, D. V. (2015). Coordinated voltage control scheme for distribution grid with on-load tap-changer and distributed energy resources in a market context. In Proceedings of 201541 th IEEE IES Annual Conference (IECON'15) IEEE.

\section{General rights}

Copyright and moral rights for the publications made accessible in the public portal are retained by the authors and/or other copyright owners and it is a condition of accessing publications that users recognise and abide by the legal requirements associated with these rights.

- Users may download and print one copy of any publication from the public portal for the purpose of private study or research.

- You may not further distribute the material or use it for any profit-making activity or commercial gain

- You may freely distribute the URL identifying the publication in the public portal 


\section{Coordinated voltage control scheme for distribution grid with on-load tap-changer and distributed energy resources in a market context}

\author{
Xue HAN and Henrik W. BINDNER \\ Technical University of Denmark \\ Frederiskborgvej 399, Roskilde, Denmark \\ Email: xueh, hwbi@elektro.dtu.dk
}

\author{
Jasmin MEHMEDALIC and David V. TACKIE \\ Danish Energy Association \\ Rosenørns Allé 9, Frederiksberg, Denmark \\ Email: jme, dvt @ danskenergi.dk
}

\begin{abstract}
The evolutionary changes in the electricity system are reshaping the system operation and control to achieve a more sustainable environment. In this transition, distributed energy resources (DERs) may introduce some problems, such as intermittent features, but could also play an important role on accommodating the intermittence and assisting system operation. A proper aggregation and coordination scheme of DER units is required to enable the flexibility provision in an economical and efficient manner. In the meanwhile, nondistruptive control of the units is essential to guarantee their long-term active participation. In addition, a newly established system should also allow and coordinate other controllable grid facilities. This paper proposes a hierarchical operating framework for DER units and controllable grid assets in a market environment. Voltage regulation as a common distributed system service is used for case study. The necessity of the coordination between DER units and the grid facilities, e.g., on-load tap-changer (OLTC), is addressed.
\end{abstract}

Keywords-Distributed energy resource (DER), coordinated control, voltage control, on-load tap-changer (OLTC)

\section{INTRODUCTION}

A sustainable energy system as the major goal in this century triggers an evolutionary transition of the energy structure as well as energy resources in the European electricity system: a growth of distributed renewable sources (e.g., wind and photovoltaic systems) and substitution of the fossil fuels by promoting electric vehicles and electric space heating [1]. In order to accommodate intermittent distributed generation and increasing electric consumption, more flexibility in both generation and consumption is needed. The flexibility from distributed energy resources (DERs) can be considered as a strong candidate, if it is observed and controlled. DERs are defined as small units, installed in the distribution network, which are capable of shifting or curtailing their production or consumption as demanded by external instances. The adjustable portion is referred to as flexibility. As the ability to control loads in response to incentives becomes more prevalent, DERs become an attractive candidate to provide system services, e.g., voltage regulation.

Different forms of voltage control solutions are proposed in the literature to mitigate negative impacts of DER integration and existing grid-operating problems [2]-[6]. [2] designed a hierarchical framework for DER management and distribution

This work is supported by Danish national project iPower - a Strategic Platform for Innovation and Research within Intelligent Electricity (SPIR). grid operation. In the framework, a sensitivity matrix is being used to describe the relation between voltage and power injection. Active power injection from the whole network and voltage boundaries are the major concerns of the design. Power injection of a small area in a lower level is exchanged with the controller on a higher level that coordinates several small areas. However, the on-load tap-changer (OLTC) is not considered in the control setup. A centralized control strategy for managing reactive power from distributed generation (DGs) is proposed in [3] to adjust voltage profiles in a medium-voltage (MV) grid. The relationship between power injection from DGs and the voltage variation given by the tap changer is derived by the sensitivity matrix between power injection and voltage variation at all nodes in the network. The objective is to maximize the reactive power injection from the controlled region. An improved OLTC local control strategy was proposed in [4], in which the considerations of network topology and loading conditions are used to support the control actions. Similar to [3], the work proposed in [5] used dynamic programming to optimize the power injection from DGs and the actions of the OLTC. [6] provided a control strategy to coordinate the action of energy storage and the OLTC in the network. The OLTC only takes care of the bus with largest voltage range compared to the reference value in the system, while the energy storage system tries to balance its own state of charge and to maintain its local voltage. The charging and discharging operations of the energy storage system smooth out the entire load profile. Therefore, less actions are trigged on OLTC during peak situations.

The listed papers provided various ways of utilizing the flexibility from DER units and the coordination between OLTC actions in the distribution network. The central entity, e.g., a distribution system operator (DSO), determines the optimal setup for both DER units and the OLTC by following their technical constraints. However, in a market context, when several entities interact with each other and trade flexibility in the market, the coordination between OLTC and DER units becomes difficult: the responsibility of different assets participating in the operation belong to different entities, and the solution should also be cost-effective and beneficial for the whole system. Therefore, the action plan of the OLTC has to be integrated with the flexibility coordination scheme, in order to make the most efficient and cost-effective use of the controllable resources in the network. In the paper, we will 
propose a control scheme to enable the coordination between the OLTC and flexibility in a market context.

The remaining part of the paper is structured as follows: Section II proposes the hierarchical control scheme in a market environment, and discusses how the control method could assist grid operation. Section III specifies the algorithm used for the coordination. It is followed by the case study in Section IV, which contains the simulation results and analysis. The paper is concluded in Section V.

\section{HIERARCHICAL AGGREGATION STRUCTURE IN MARKET CONTEXT}

A hierarchical aggregation and control structure for the DER units and flexibility management is proposed in [7] and further developed in [8]. The aggregation is based on the physical features of DER units, i.e., electric location in this work. The aggregated information contains real and reactive power capability and pseudo cost. By aggregating the available and distributed small-scale resources in the grid, a systemscale service can be achieved by allowing the aggregation and dispatch of flexibility from DER units.

\section{A. Proposed voltage control structure}

The previous published work in [7] described the basic hierarchical control structure and the modular functions of different roles in the structure. The entities and their responsibilities are further clarified in [8] in a market context. Fig. 1 shows how different stakeholders interact with each other, and how the information, including control commands, is transferred. In the figure, the aggregator is the entity who aggregates and coordinates the flexibilities from various DER units, as well as presents their service-provision capabilities to the grid. The DER units are owned by customers, and the access to partial or full information about the units is given to the aggregator. The DSO owns the grid assets and maintains their safe operation. OLTCs as one type of direct controllable devices are foreseen to be installed and operated in the MV grid to maintain the voltage level in the distribution network. In a liberalized market context, the DSO and aggregators have distinct responsibilities, information accesses, and control accesses to different sources in the system. The interactions between the DSO and aggregators in terms of trading the flexibility for different types of grid services and technical implementation requires a new market place with integrated communication structure. In iPower [9], a Danish research and innovation project, a FLExibility Clearing House (FLECH) is proposed to enable transparent trading of flexibility and grid support services, especially on the distribution grid operation [10]. The market actions and information flow have to go through FLECH to ensure fair trading for aggregators and transparency in settlement.

Flexibility from DER units are usually in small scale. In addition, there are large amounts of units located in a large area and with different operating principles. Therefore, the flexibility control scheme has to face the aggregation challenges provided by the nature of DER units and has to be as robust as conventional solutions. In our previous work presented in [7], a hierarchical aggregation and control structure is proposed (see Fig. 2). The aggregation is based

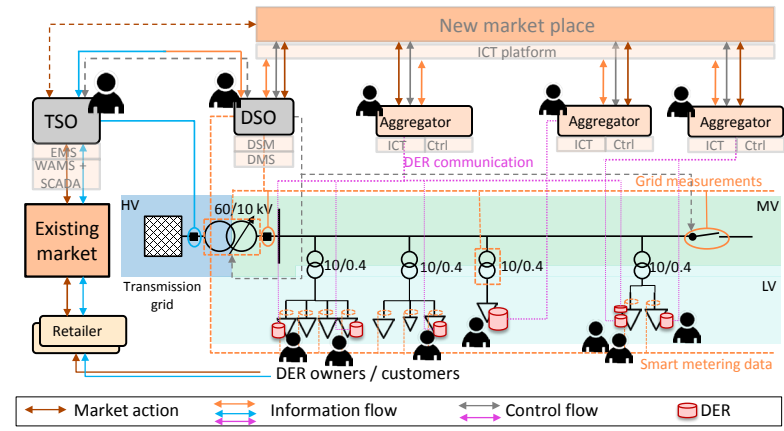

Fig. 1: Market based distribution grid operation with DER integration.

on the physical features of DER units, i.e., location, and other properties. DERs are interfaced with the control system through the unit controller (UC). The detailed information regarding one specific DER unit is hidden under the UC. DERs are dynamically grouped under a local controller (LC), which handles the primary aggregation of the flexibility from units and optimally dispatches the local resources based on the setpoints from the supervisory controller (SC). The supervisory controller (SC) handles the global information and makes optimal decisions for the network. The hierarchy is built in a flexible and fault-tolerant manner where the roles as LCs or the SC in different levels of the hierarchy are elected among the participating controllers of available physical units [11].

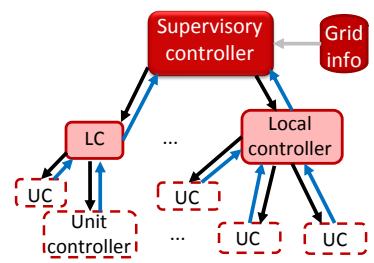

(a) Control hierarchy

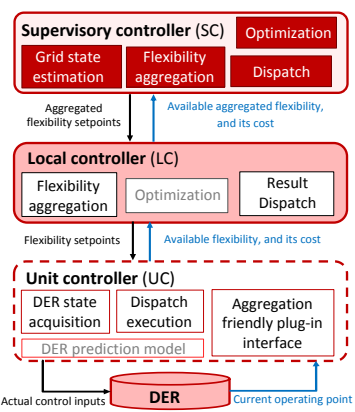

(b) Roles
Fig. 2: Sketch map of the hierarchical controller.

In a market context, the modular functionalities within the SC can be further specified by whom they belong to based on the responsibility and the ownership of resources, as shown in Fig. 3. The map between the geographical location and electrical locations of certain DER units is used to translate the information between the DSO and aggregators. The DSO finds the optimal operational conditions for the available DER units and controllable grid devices. Aggregators optimize their own portfolios of flexibility by following the decisions generated in the market. The aggregation scheme makes sure that the goal from the DSO can be achieved by allocating the commands to each controllable resource in the system.

\section{B. Compatibility issue with controllable grid assets}

The traditional OLTC operates autonomously according to a predefined rule by tracking the grid measures, e.g., voltage 


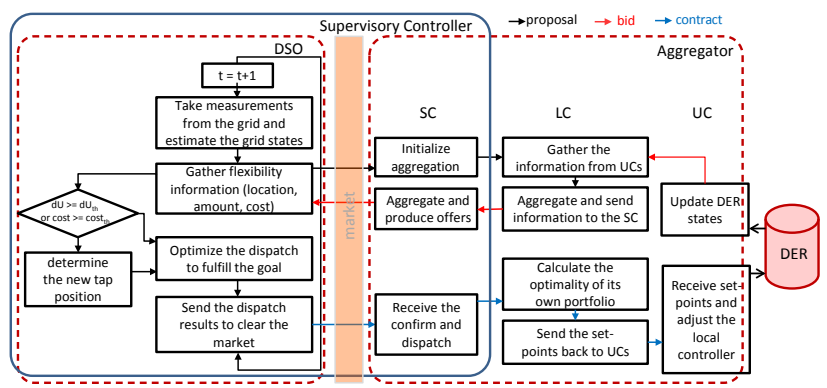

Fig. 3: The flow chart of the proposed coordination control structure. The modular functions within the technical SC is separated to the DSO functions and aggregator functions. All the information exchange is through the market between the DSO and the aggregator.

measurement at the secondary side of the transformer [12]. If the flexibility is used to regulate voltage in the network at the same time, overcompensation may happen if the actions are made in the meanwhile. Since the OLTC is the most direct way to regulate voltage, it is valuable for the DSO to utilize both the OLTC and the flexibility to obtain more efficient and cost-effective solutions than using either of two alone. Therefore, coordination between the OLTC and the flexibility management system is essential to achieve. As part of the grid assets owned by the DSO, the participation of the OLTC does not have to go through the market together with the flexibility from DER units. It is reasonable to manage the actions of the OLTC when the DSO is submitting the requirements to the market within its own management system. However, in a market context, the DSO will evaluate the value of changing the tap position with activating the flexibility from DER units. Therefore, adding the value or pseudo cost for the OLTC actions in the decision making process is an easy and reasonable way to integrate both resources.

\section{COORDINATION ALGORITHM}

In this paper we will focus on how the OLTC is coordinated with flexibility dispatch within the DSO's dispatch module. The flexibility dispatch can be formulated as a multiobjective optimal power flow problem. We uses photovoltaic (PV) panels, and electric vehicles (EVs) to analyse the impacts of DER units with diversified characteristics. The dispatch handles faster variations and smooths the peaks with shorter durations, while the tap changing captures the longer term variations and reduces the overall cost for maintaining the system operation. Two indices are taken into consideration to trigger the tap changing: voltage level at the most critical point in the network, and the overall cost of utilizing the flexibility in the system. The set-points for DER units are dispatched every 1 minute, and the optimal tap position will be determined if either condition triggers.

\section{A. Optimal power flow problem}

Both system operational constraints and the physical constraints from DER units are considered in the optimization problem formulation. The system variable set is defined as $x=\left[\triangle P^{+}, \triangle P^{-}, \triangle Q^{+}, \triangle Q^{-}\right]^{T} . \triangle S_{\text {flex }}^{*}$ is the optimal set for the system. Its relationship with the system variables, $\frac{d S}{d x}$, can be derived as:

$$
\triangle S_{\text {flex }}=\left[\begin{array}{cccc}
-1 & 1 & 0 & 0 \\
0 & 0 & -1 & 1
\end{array}\right] \times x
$$

where, $\triangle P^{+}, \triangle P^{-}, \triangle Q^{+}, \triangle Q^{-}$are the active and reactive flexible power production on both directions, i.e., up and down regulation.

The objectives of the problem are to minimize the total active power loss in the network and to minimize the total pseudo cost $c_{u}$ from DER units. The total active power loss is given by the multiplication of the sensitivity matrix $\frac{\partial P_{\text {loss }}}{\partial S}$ and $\triangle S_{\text {flex }}$. The detailed derivation can be found in [7]. The weighting factors can be customized to fulfil the needs from the DSO. Sensitivity analysis has been made in [7].

$$
\min \sum^{i}\left(c_{i} \cdot x+\frac{\partial P_{\text {loss }}}{\partial S} \cdot \frac{d S}{d x} x\right)
$$

where, $i$ is one node in the system, $c_{i}$ is the aggregated cost value at node $i$ given available flexibility under the node [7].

1) grid constraints: The modified voltage magnitude at node $i, U_{i}=U_{0, i}+\triangle U$ should be within the hard boundary:

$$
U_{i, \text { min }}-\triangle U_{t o l} \leq U_{0, i}+\frac{\partial U}{\partial S} \triangle S_{f l e x} \leq U_{i, \max }+\triangle U_{t o l}
$$

where, $U_{0, i}$ is the estimated voltage at the next time stamp given the default inputs, $\frac{\partial U}{\partial S}$ provides the sensitivity between the power injection and voltage at every node [7], $U_{i, \max }$ and $U_{i, \min }$ are maximal and minimal limit value allowed in system operation, and $\triangle U_{t o l}$ gives a small tolerance region for the voltage to overpass, so that the controller could identify the critical voltage situations.

In addition, we also introduce long term moving average, $\overline{U_{i}}(t)$, as the reference value on each node that the voltage need to follow:

$$
\overline{U_{i}}(t)=(1-\alpha) \overline{U_{i}}(t-1)+\alpha U_{i}(t)
$$

where, $0 \leq \alpha \ll 1$. The difference between the moving average value and the actual voltage magnitude needs to be minimized as an additional part of the objective. The introduction of the moving average could avoid large variations brought by the optimization and provide a moderate reference value to avoid over compensation.

The apparent power loss is strongly related to the thermal limit of the cable. Therefore, the apparent power loss on each line is also constrained in the problem:

$$
-S_{\text {loss }}^{0} \leq \frac{\partial S_{\text {loss }}}{\partial S} \triangle S_{\text {flex }} \leq S_{\text {loss }}^{\max }-S_{\text {loss }}^{0}
$$

where, $S_{\text {loss }}^{0}$ is the estimated loss on each line at the next time stamp given the default inputs (the injected power from DER when no control is deployed), $S_{\text {loss }}^{\max }$ is the absolute maximal power loss allowed on each line, $\frac{\partial S_{\text {loss }}}{\partial S}$ is the linear sensitivity matrix derived on the estimated operating point. 


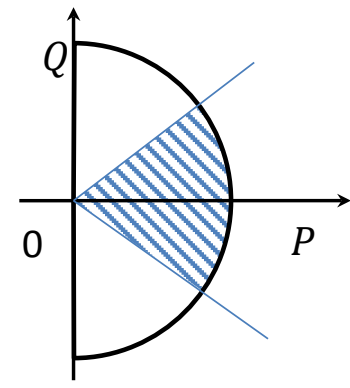

Fig. 4: Illustration of physical constraints of PV units. The shadow area is the flexibility region. The circle shows the capacity constraint, while two lines give the power factor constraints.

2) physical constraints of PVs: The default inputs from $\mathrm{PVs}$ are full potential active power production without curtailment and zero reactive power injection. Consequently, the flexibility from PVs is reactive power injection or absorption and active power production. There are two constraints: power factor $\theta_{P F}$, and the inverter capacity limit $S_{P V, \max }$. They are illustrated in Fig. 4. For a PV unit, the flexibility $\triangle P^{-}$and $\triangle Q^{+,-}$can be given as

$$
\begin{gathered}
0 \leq \triangle P^{-}(t) \leq P_{P V}(t) \\
0 \leq \triangle Q^{+,-}(t) \leq\left(P_{P V}(t)-\triangle P^{-}(t)\right) \cdot \tan \left(\theta_{P F}\right) \\
\left(\left(P_{P V}(t)-\triangle P^{-}(t)\right)^{2}+\triangle Q^{+,-}(t)^{2}\right) \leq S_{P V, \text { max }}^{2}
\end{gathered}
$$

The pseudo cost of the flexibility from a PV unit is determined by the amount that is available. The more the PV unit produces at a certain time instant, the cheaper the cost per unit is. The active power curtailment is much more costly than reactive power support.

3) physical constraints of EVs: The EVs are only considered to be charged at home and no Vehicle-to-grid service is considered. The default condition for an EV is it will be charged if the EV is at home and its battery is not full. In order to fulfil the need of the owners, the flexibility an EV can provide is to postpone the charging only if the battery can still be fully charged before the next time the owner needs it. The constraints are expressed as:

$$
\left\{\begin{array}{cl}
0 \leq \triangle P^{-}(t) \leq P_{E V, r a t e} & , \text { if } t_{a l}<t_{c} \\
\triangle P^{-}(t)=0 & , \text { if } t_{a l} \geq t_{c}
\end{array}\right.
$$

when the the EV is at home and its battery is not fully charged. $P_{E V \text {, rate }}$ is the rated charging power of an EV. $t_{a l}$ is the remaining available charging duration before the EV departs, while $t_{c}$ is the minimal required duration before the EV can be fully charged.

The pseudo cost for offering the flexibility is proportional to the ratio between $t_{c}$ and $t_{a l}$. The cost is comparably lower than the power curtailed from PV and is higher than the reactive power compensation.

\section{B. Coordination with OLTC}

The optimal tap position needs to be chosen simultaneously when the optimal flexibility dispatch is made, so that the DSO can find the most cost effective way to manage the controllable resources in the system. In addition to sensing the voltage level in the network, total cost of flexibility is also added as another metric for the purpose of best resource allocation and cost effectiveness. Once one of the metrics are violated during the operation, the DSO will try to find the best tap position by finding the minimal cost through solving multiple optimization problems with all possible tap positions.

In our work, the OLTC is modelled as a series impedance with the capability to change the voltage ratio on two sides of it. Given different voltage set-points at the point of common coupling, it is possible for the controller to derive the best dispatching results accordingly. By comparing the results, the best tap position can be determined. The corresponding dispatch result will be sent to the aggregator to find the optimal set-points of individual DER units. The tap changing decision process can be triggered by either the voltage violation or the overall cost higher than a threshold value determined by the DSO. The threshold value can be determined based on the investment and life cycle of the OLTC.

\section{CASE STUDY}

\section{A. Grid and load models}

A tailored IEEE distribution 4-bus feeder [13] (see Fig. 5) is used for the simulation. The length of the feeder is extended in order to correspond with the average length of a Danish MV distribution feeder. To have a better understanding of both MV and LV operation situation, a detailed LV feeder as shown in Fig. 6 replaces the load on the last node in the MV feeder. The length of the MV feeder is approximately $10 \mathrm{~km}$, and it is $0.8 \mathrm{~km}$ for the LV part. The average $\mathrm{R} / \mathrm{X}$ ratios of the cables in the MV and LV grid are approx. 2 and 3 respectively. There are in total $51 \mathrm{EVs}$ and $138 \mathrm{PV}$ panels in the system. 6 types of customers are considered in the network. Most of them are residential. The peak load is approx. 3.5 MW. More detailed information about load models can be found in [8]. Fig. 7 shows the daily load profile and its different portions at the last node in the MV network. The OLTC is installed at the secondary side of the transformer on primary substation between HV and MV. There are 7 tap positions for the OLTC, which evenly separate the range between $+5 \%$ and $-5 \%$ of the nominal value to provide a better system performance. In the scenarios without the OLTC, the voltage at the point of common coupling is set to 1.04 p.u. as a constant number.

\section{B. Scenarios and simulation results}

Four scenarios are considered in the analysis:

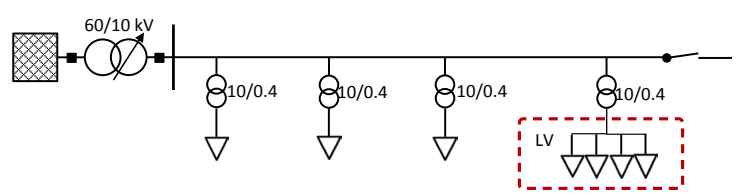

Fig. 5: Single line diagram of the MV distribution feeder. The LV feeder is replaced by the detailed LV Danish network. 


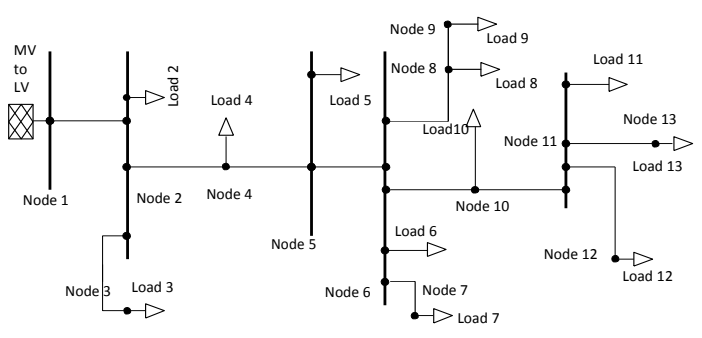

Fig. 6: A Danish LV feeder.

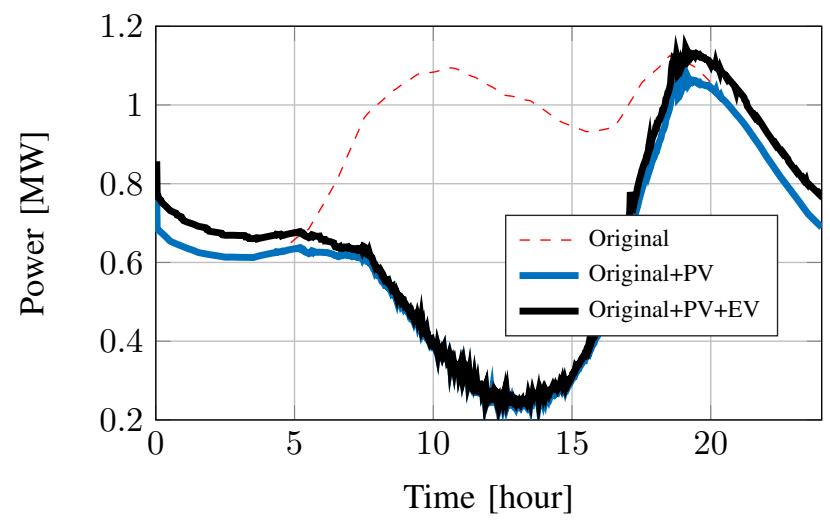

Fig. 7: The daily load profile at the last node in the MV network.

- $\quad$ Sc1 - reference In this scenario, no advanced control is applied. The system operates as a passive network with connection to DER units.

- $\quad$ Sc2 - OLTC The autonomous OLTC application is used in this scenario. The OLTC changes the tap positions according to the voltage on the most critical node in the network following a predefined rule.

- $\quad$ Sc3 - controller The flexibility controller is applied in this scenario, but no OLTC is available. The system operates with the assistance of flexibility from DER units.

- $\quad$ Sc4 - ctrl+OLTC The coordinated control scheme is used to coordinate the behaviours of the OLTC and the DER units.

- Sc5 - ctrl+OLTC The cost threshold of OLTC is set as $50 \%$ of the original value, so that the tap changing decision is more sensitive to the overall cost of activating flexibility services.

The simulating period is 5 days with 1 minute resolution. LCs are located in all the nodes in the system where there are DERs to be aggregated. The aggregating zones presented in [8] are not applied in this work, since they are not very relevant to the focus in this work.

Fig. 8 shows the distribution of the voltage magnitude for the whole simulation period in different scenarios. Table I summarizes several key numbers in the simulations. It can be seen that by having the OLTC in the network, the difference between the maximal and minimal voltage magnitudes decreases significantly. Since during the peak loading period, there is not very much flexibility available in the system, the OLTC contributes very much on bringing the voltage up. The

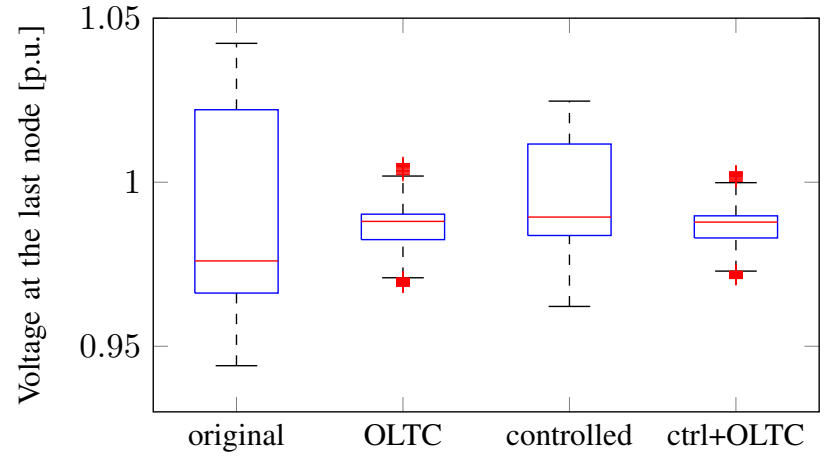

Fig. 8: Boxplot of the voltage on the most critical node in the network in different scenarios.

TABLE I: Summary of simulation results (The values are presented as comparable numbers to "1"). The number of actions is accumulated for one day.

\begin{tabular}{c|ccc}
\hline & $\begin{array}{c}\text { Total } \\
\text { cost }\end{array}$ & $\begin{array}{c}\text { Overall } \\
\text { loss }\end{array}$ & $\begin{array}{c}\text { No. of } \\
\text { actions }\end{array}$ \\
\hline \hline Sc2 & - & 1 & 9 \\
Sc3 & 1 & 0.86 & - \\
Sc4 & 0.91 & 0.82 & 4 \\
Sc5 & 0.76 & 0.83 & 7 \\
\hline
\end{tabular}

voltage is not limited into a very narrow range in $\mathrm{Sc} 3$ because the overall cost and power loss are also objectives considered in the optimization. A tighter voltage control requires higher cost and may lead to higher losses as well. The figure also shows that the voltage range is further narrowed down in $\mathrm{Sc} 4$, by coordinating the tap changing and the flexibility dispatch. It can also be seen from the table that the number of OLTC actions can be reduced by integrating flexibility management into the operational control scheme. Both cost and system losses can be reduced by having the DER reacting in the system. By reducing the cost threshold value for the OLTC, the OLTC acts more frequently and the total cost is reduced accordingly. This scenario also indicates that by installing the OLTC in the system, there will be a risk that the flexibility may not be used so often for voltage regulation due to the cost issue. By comparing the losses in different scenarios, it can be identified that the reactive power compensation by the DER units reduces the current flowing in the network, and thus reduces the overall system losses. Flexibility from DERs is a more efficient way to reduce losses.

Fig. 9 shows the comparison of load profiles among Sc1, Sc3 and Sc4. It can be seen from the figure that no PV production is curtailed in any of the scenarios. In addition, the EV charging actions are not postponed significantly in Sc4 as in Sc3. It is because during Hour 22 - Hour 24, the OLTC taps the voltage to a higher value, so that the controller does not require much flexibility to be shifted to later period. During Hour 5 - Hour 7, since there is not much flexibility available, and the cost for postponing the charging is high, the OLTC taps the voltage again to avoid higher cost. Therefore, several more EVs could charge earlier so that the battery can be fully charged in the morning for the next commute.

Fig. 10 illustrates the active and reactive power curve for a PV unit in the network. The yellow curve shows the reactive power curve in $\mathrm{Sc} 3$, and the orange curve shows the reactive 


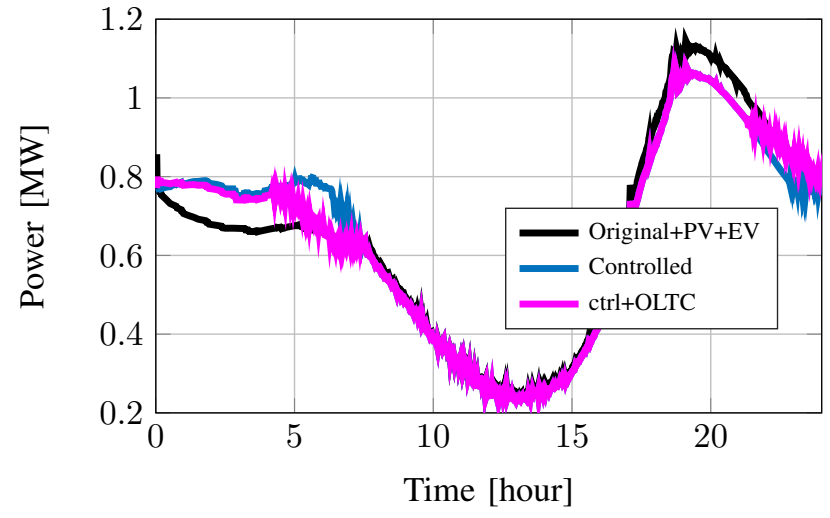

Fig. 9: The load profiles at the last node in the MV network in different scenarios.

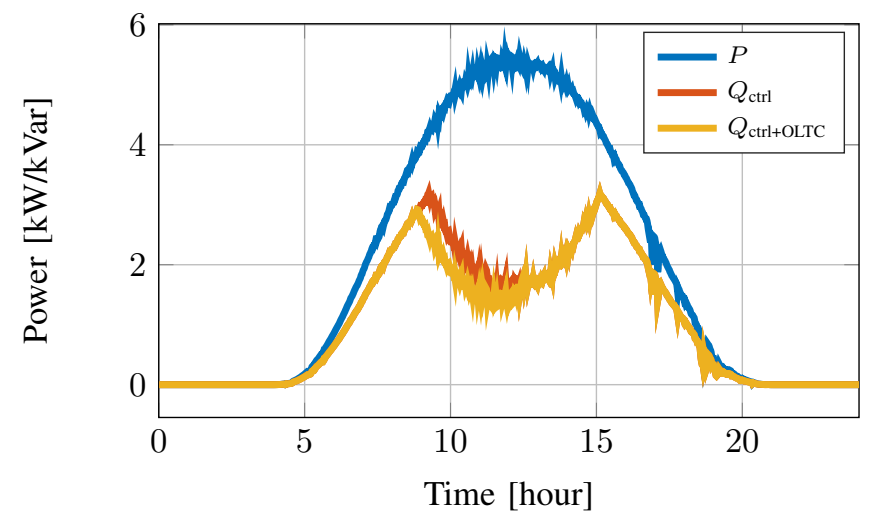

Fig. 10: The PV production curves in different scenarios.

power injection in $\mathrm{Sc} 4$. Since the reactive power is limited by the active power production at a certain point in time, during Hour 5 - Hour 10 and Hour 15 - Hour 20, the unit cannot inject more reactive power. Therefore, it produces as much as it could. The changes of tap position cannot be reflected in the curve. During Hour 10 - Hour 15, the PV production gets close to the load. Therefore, the reactive power compensation is reduced. The difference of the reactive power profile in Sc3 and Sc4 occurs during Hour 10 - Hour 13, because in Sc4, a higher tap position brings up the system voltage in $\mathrm{Sc} 4$, and less reactive power is needed.

\section{CONCLUSION}

This paper investigates how the OLTC can serve for voltage regulation together with flexibility management system, and proposes a coordinated control scheme to integrate both types of control resources together for grid operation. In a market context, by comparing the values of taping the OLTC and activating the flexibility, the control system could make an optimal decision for the DSO given all available resources.

In this paper, a two voltage level network is used to simulate and analyse the system operation without and with proposed control scheme. It also presents the simulation results showing that the coordinated control scheme could reduce the potential cost for the system as a whole. In addition, a better voltage profile could be obtained by activating all available resource than only utilizing one type. The OLTC could keep the voltage in an preferable range when there is no flexibility available in the network, while the flexibility activation avoid frequent changes of tap potions of the OLTC. However, the sensitivity analysis of the cost threshold value for the OLTC shows that the performance of the system is very dependent on the estimated cost of one tap changing. The needs for flexibility activation on voltage regulation may disappear if either flexibility is too costly or the cost of OLTC is very cheap.

One radial feeder is considered in this paper under the primary substation. However, in reality, multiple feeders may be fed by one primary substation. Under the circumstances when different types of customers and different amounts of DGs are connected in different feeders, the voltage regulation capability one OLTC could provide is limited. This problem will be tackled afterwards.

\section{REFERENCES}

[1] The 2020 climate and energy package. European Commission, Last retrieved 20.10.2014. [Online]. Available: http://ec.europa.eu/clima/ policies/package/index_en.htm

[2] M. Bayat, K. Sheshyekani, and A. Rezazadeh, "A unified framework for participation of responsive end-user devices in voltage and frequency control of the smart grid," Power Systems, IEEE Transactions on, 2014, early access.

[3] A. Keane, L. F. Ochoa, E. Vittal, C. J. Dent, and G. P. Harrison, "Enhanced utilization of voltage control resources with distributed generation," Power Systems, IEEE Transactions on, vol. 26, no. 1, pp. 252-260, 2011.

[4] F. A. Viawan, A. Sannino, and J. Daalder, "Voltage control with onload tap changers in medium voltage feeders in presence of distributed generation," Electric power systems research, vol. 77, no. 10, pp. 13141322, 2007.

[5] Y.-J. Kim, S.-J. Ahn, P.-I. Hwang, G.-C. Pyo, and S.-I. Moon, "Coordinated control of a dg and voltage control devices using a dynamic programming algorithm," Power Systems, IEEE Transactions on, vol. 28, no. 1, pp. 42-51, 2013.

[6] X. Liu, A. Aichhorn, L. Liu, and H. Li, "Coordinated control of distributed energy storage system with tap changer transformers for voltage rise mitigation under high photovoltaic penetration," Smart Grid, IEEE Transactions on, vol. 3, no. 2, pp. 897-906, 2012.

[7] X. Han, A. M. Kosek, O. Gehrke, H. W. Bindner, and D. Kullmann, "Hierarchical control architecture to activate distributed energy resources' services: Voltage control as an application," in $T \& D$ Conference and Exposition, 2014 IEEE/PES, 2014.

[8] X. Han, H. Bindner, J. Mehmedalic, and D. Tackie, "Hybrid control scheme for distributed energy resource management in a market context," in PES General Meeting, Conference \& Exposition, 2015 IEEE. IEEE, 2015.

[9] iPower - Strategic Platfor for Innovation adn Research in Intelligent Power . (2015). [Online]. Available: http://ipower-net.dk/

[10] K. Heussen, D. E. M. Bondy, J. Hu, O. Gehrke, and L. H. Hansen, "A clearinghouse concept for distribution-level flexibility services," in Innovative Smart Grid Technologies Europe (ISGT EUROPE), 2013 4th IEEE/PES. IEEE, 2013.

[11] A. M. Kosek, O. Gehrke, and D. Kullmann, "Fault tolerant aggregation for power system services," in Intelligent Energy Systems (IWIES), 2013 IEEE International Workshop on. IEEE, 2013, pp. 107-112.

[12] C. Gao and M. A. Redfern, "A review of voltage control techniques of networks with distributed generations using on-load tap changer transformers," in Universities Power Engineering Conference (UPEC), vol. $31,2010$.

[13] IEEE PES 4-bus Distribution Test Feeder. (2015). [Online]. Available: http://ewh.ieee.org/soc/pes/dsacom/testfeeders/index.html 